\title{
Executive Function in SLI: Recent Advances and Future Directions
}

\author{
Leah L. Kapa ${ }^{1}$ - Elena Plante ${ }^{1}$
}

Published online: 25 June 2015

(C) Springer International Publishing Switzerland 2015

\begin{abstract}
This paper provides a review of recent research on executive function abilities in children with specific language impairment (SLI). Across several studies, children with SLI are reported to perform worse than typically developing peers on measures of sustained attention, working memory, inhibition, and attention shifting. However, few studies have considered multiple executive function components simultaneously and even fewer have examined the underlying relationship between executive function deficits and impaired language acquisition. We argue that in order to fully understand the nature of executive function deficits in SLI, the field must move past simply identifying weaknesses to instead test models of executive function development and explore the nature of the relationship between executive function and language. Future research directions are recommended in order to achieve these goals.
\end{abstract}

Keywords Specific language impairment - Executive function · Working memory · Attention · Inhibition · Attention shifting $\cdot$ Cognitive development

\section{Introduction}

Although specific language impairment (SLI) is defined by disordered acquisition of language in the absence of hearing

Leah L. Kapa

leahkapa@email.arizona.edu

Elena Plante

eplante@email.arizona.edu

1 Department of Speech, Language, and Hearing Sciences, University of Arizona, 1311 E. 2nd Street, P.O. Box 210071,

Tucson, AZ 85721-0071, USA impairment, neurological damage, cognitive impairment, and concomitant behavioral or emotional disorders [1], researchers have questioned whether children with SLI are accurately characterized by these exclusionary criteria. In addition to language impairment, nonlinguistic deficits have been identified among children diagnosed with SLI including in motor control [2, 3], response speed [4], and memory [5, 6]. Many children with SLI also demonstrate cognitive deficits when compared to typically developing peers on measures of both verbal and nonverbal cognition [7]. Indeed the nonverbal IQ diagnostic criterion of SLI has been at the center of ongoing debate within the field [8-12].

In light of the evidence that children with SLI have weaknesses that are not restricted to language, domain-general accounts of the disorder have been proposed. Perhaps most recently, Ullman and Pierpont [13] introduced the Procedural Deficit Hypothesis in which disordered language development stems from an aberration in the neural network underlying procedural memory, which leads to the hallmark grammar impairments as well as additional nonlinguistic deficits in cognition and motor skills that have been reported among children with SLI. The primary evidence presented in support of this hypothesis is differences on serial reaction time tasks by children with SLI compared to their typically developing peers [14]. This particular theory was preceded in time by other theories that also posited that more general deficits could account for language deficits. The Generalized Slowing Hypothesis [15] suggests that children with SLI are slower to process stimuli across both linguistic and nonlinguistic domains $[15,16]$. This slowed processing is assumed to disrupt language acquisition because crucial language learning operations like parsing the speech stream and extracting words/ morphemes are time-dependent. Earlier still, the Generalized Representational Deficit [17] proposed that the language deficits of children with SLI were part and parcel of an 
overarching problem with the representation of information in general. This theory found support from studies that demonstrated subtle but reliable nonverbal cognitive deficits in the visuo-spatial domain $[18,19]$, hierarchical planning [20], and haptic recognition [21].

Each of these theories represents a decades-long attempt to find a single base deficit that explains the range of surface signs that present in SLI (a "Grand Unification Theory" of SLI). Over time, these attempts have waxed and waned as initial evidence in support of the theory became available, inevitably followed by counter-evidence. For example, several studies have failed to find uniform deficits for serial reaction time learning in SLI or attributed the locus of the deficit to factors other than procedural learning per se [22-24]. Failures to link slow reaction times to language deficits undermined the Generalized Slowing Hypothesis [25]. Other theories (e.g., the General Representational Deficit) have undergone a reconceptualization. For example, the co-occurrence of discrete verbal and nonverbal deficits may be the functional consequence of both right and left hemisphere involvement in SLI [26] rather than signaling an overarching representational deficit.

The regular appearance of new Grand Unification Theories in SLI provides an object lesson for our consideration of executive function in this paper. It has been relatively easy over the years to find deficits outside of the language deficits that have classically defined SLI. It has been substantially more difficult to come to an understanding of the role of these nonlinguistic deficits in the overall presentation of the disorder. Research studies in these areas often rely on correlational methods rather than the types of experimental manipulations that would establish causal connections. Conversely, it is not necessary for an underlying cognitive deficit to account for impaired language development in order for it to be important in the overall constellation of the disorder. For example, slow processing speed during the period of language acquisition may not itself give rise to SLI, but slow processing speed is not likely to facilitate language processing for those who do develop SLI. Therefore, whether nonlinguistic deficits constitute causal, contributing, or co-occurring signs, understanding the broader profile of SLI is important. However, a full understanding of the SLI profile will not only include a list of verbal and nonverbal deficits, but whether and how these deficits inter-relate.

\section{Executive Function}

In support of domain-general accounts of SLI, there is increasing evidence that children with SLI demonstrate deficits on both verbal and nonverbal measures of executive function. Executive function comprises higher-order cognitive skills that control and coordinate attention, thought, and action. These skills are largely associated with prefrontal cortex involvement, and they show inverted U-shaped development across the lifespan as they slowly develop through childhood $[27,28]$ to eventually peak in young adulthood followed by a decline associated with aging in later adulthood [29]. Executive function abilities are crucial for regulating attention and behavior and are predictive of academic success [27, 30-32].

Conceptualizations of executive function range from a unitary construct $[33,34]$ to a set of independent components [35, 36]. Many recent models combine these approaches into integrative models, which posit that executive function comprises a set of separable, but related cognitive components. The most widely applied integrative model of executive function comes from Miyake et al. [37] in which adult participants' performance on a battery of executive function measures was analyzed using factor analysis to delineate separable components within executive function. This led to the conclusion that executive function can be separated into three components: updating (or working memory), inhibition, and shifting attention, which are unique, but moderately correlated.

\section{Developmental Integrative Framework Model}

Garon et al. [38] applied Miyake and colleagues' [37] integrative model of executive function to the development of these skills from infancy through the preschool period in their developmental integrative framework model. Based on the order in which different executive function components emerge, Garon et al. posit a hierarchical relationship between components. Specifically, Garon and colleagues' developmental integrative framework model suggests that the first relevant skill to develop is sustained attention, which, although not typically considered an executive function, is a basic cognitive skill that forms the basis for the development of more complex executive function skills. The next component to emerge is working memory, followed by inhibition, and then attentional shifting. Working memory is the ability to retain and manipulate new information over a brief period of time. Inhibition is the ability to ignore distracting information or avoid making a prepotent, but incorrect response. Finally, attentional shifting, or cognitive flexibility, refers to the process of moving attention between different stimuli, stimulus properties, or tasks.

Within the developmental integrative framework model, it is assumed that later-developing skills also require coordination between earlier-developing abilities. For example, a working memory task also requires sustained attention, whereas successful completion of an attentional shifting task requires sustained attention, working memory, and inhibition. Garon et al. [38] created their model based upon the development of executive function among children with typical development, but it has interesting implications for cases of disordered executive function development. Based on the hierarchical nature of the model, disruptions in early developing skills would logically lead to deficits in the laterdeveloping skills that they underlie. In other words, problems 
with the development of lower-level components would have cascading effects on higher-level components.

Executive function is interesting to consider among children with impaired language because of the bidirectional relationship between these cognitive skills and language development. For example, language in the form of self-directed or internalized speech is an effective strategy for mediating responses on executive function tasks. When preschoolers are encouraged to produce relevant language during executive function tasks, performance improves [39, 40]. Likewise, when adults and older children are prevented from using language through articulatory suppression, executive function performance declines $[41,42]$. In the opposite direction, individuals' executive function abilities predict their success in learning new vocabulary $[43,44]$ and interpreting ambiguous sentences [45]. Additionally, individuals with impaired executive function due to developmental disorder (e.g., Attention Deficit Hyperactivity Disorder) or aging have reduced language abilities $[46,47]$. Children with SLI present a fascinating case because their disordered language may lead to executive function deficits or, conversely, may be a result of underlying executive function deficits.

\section{Executive Function in Children with SLI}

Researchers have compared the performance of children with SLI to their peers with typical language development on a variety of measures assessing each of the four executive function components included in the developmental integrative framework model [38]. Sustained attention has been assessed via continuous performance tasks in which children must respond to the presence of a target stimulus (visual or verbal) while withholding responses to non-target stimuli across a large number of trials, the majority of which are non-target trials $[48,49]$. Researchers have used span tasks that require children to hold information in mind while simultaneously performing a mental operation to measure working memory. For example, verbal working memory has been compared between children with and without SLI using a backward digit span task that requires children to repeat lists of digits in the reverse order of presentation [50-54]. Tasks that require children to withhold responding, such as the stop signal task [55] and a go/no-go task [56], have been employed to assess inhibition abilities among children with SLI. Finally, shifting abilities of children with SLI have been compared to children with typical language development using a card sorting task [57] and a trail making task [58•]. In the card sorting task, children must switch attention between different stimulus properties (color versus shape), and the trail making task requires children to connect dots in order alternating between numbers and letters (e.g., a-1-b-2-c).

The results of recent research comparing executive function components between children with and without
SLI are summarized in Table 1. For the purposes of this summary, measures are broadly divided into verbal or nonverbal categories based on whether or not tasks included verbal stimuli and/or responses. Based on these recent studies, there is converging evidence that children with SLI have deficits on verbal working memory. There is also fairly consistent evidence that these children are worse than language-typical peers on measures of nonverbal inhibition and both verbal and nonverbal sustained attention. Indeed, deficits in verbal and nonverbal sustained attention for children with SLI have been supported by the outcomes of a meta-analysis [59]. However, other skills, such as nonverbal working memory, have conflicting results with some researchers finding evidence of an SLI deficit and others reporting equivalent performance between children with SLI and those with typical language development. Despite the inconsistent outcomes among individual studies, Vugs et al. [60••] conducted a meta-analysis and concluded that the majority of evidence indicates that children with SLI perform worse than typical peers on measures of visual working memory. For the remaining components, shifting (both verbal and nonverbal) and verbal inhibition, there is too little research to reach a conclusion regarding how these skills may be affected in SLI.

Although many researchers have laid the groundwork by comparing executive function between children with SLI and children with typical language development, there is more work to be done to understand both the nature of executive function in children with SLI and the relationship between executive function and their language deficits. First of all, with a handful of notable exceptions $[58 \bullet, 61 \bullet, 62,63 \bullet, 64 \bullet]$ previous studies have considered executive function components in isolation. Therefore, little data are available to make withinsubject comparisons for different components of executive function, making it difficult to discern how these skills relate to one another within the SLI population. As previously mentioned, integrative frameworks of executive function based on typically developing children and adults assume that executive function components are dissociable, but correlated in individuals. However, it is unclear whether an integrative model accurately describes executive function among children with SLI.

A second unresolved issue in this area is the directionality of the relationship between executive function and language development in children with SLI. The question remains open as to whether executive function deficits may contribute to disordered language acquisition, or vice versa, if impaired language abilities impede executive function development. Although this issue has not been resolved, a small number of recent studies have addressed this question.

Lidstone et al. [42] tested the role of language in executive function performance among children with SLI 
Table 1 Summary of recent studies of executive function in children with SLI

\begin{tabular}{|c|c|c|c|c|c|}
\hline \multirow[t]{2}{*}{ EF component } & \multirow[t]{2}{*}{ Authors } & \multicolumn{2}{|c|}{ Participants } & \multicolumn{2}{|l|}{ Results } \\
\hline & & SLI & TD & Nonverbal & Verbal \\
\hline \multirow[t]{7}{*}{ Sustained attention } & Spaulding et al. [48] & $23(4 ; 10)$ & $23(4 ; 10)$ & $\mathrm{SLI}=\mathrm{TD}$ & $\mathrm{SLI}<\mathrm{TD}$ \\
\hline & Finneran et al. [49] & $13(5 ; 2)$ & $13(5 ; 3)$ & $\mathrm{SLI}<\mathrm{TD}$ & - \\
\hline & Dispaldro et al. [78] & $22(6 ; 3)$ & $22(6 ; 2)$ & $\mathrm{SLI}<\mathrm{TD}$ & - \\
\hline & Duinmeijer et al. [61•] & $38(7 ; 4)$ & $38(7 ; 11)$ & $\mathrm{SLI}<\mathrm{TD}$ & - \\
\hline & Montgomery et al. [79] & $26(8 ; 5)$ & $26(8 ; 2)$ & - & $\mathrm{SLI}<\mathrm{TD}$ \\
\hline & Montgomery [80] & $36(8 ; 6)$ & $36(8 ; 3)$ & - & $\mathrm{SLI}<\mathrm{TD}$ \\
\hline & Marton et al. [63•] & $22(12 ; 3)$ & $22(12 ; 6)$ & $\mathrm{SLI}<\mathrm{TD}$ & - \\
\hline \multirow[t]{12}{*}{ Working memory } & Vugs et al. [52] & $58(4 ; 9)$ & $58(4 ; 11)$ & $\mathrm{SLI}<\mathrm{TD}$ & $\mathrm{SLI}<\mathrm{TD}$ \\
\hline & Petruccelli et al. [73] & $24(5 ; 3)$ & $32(5 ; 3)$ & $\mathrm{SLI}=\mathrm{TD}$ & $\mathrm{SLI}<\mathrm{TD}$ \\
\hline & Ziethe et al. [54] & $19(5-6)^{\mathrm{c}}$ & $25(4-6)^{\mathrm{c}}$ & - & $\mathrm{SLI}<\mathrm{TD}$ \\
\hline & Duinmeijer et al. [61•] & $38(7 ; 4)$ & $38(7 ; 11)$ & - & $\mathrm{SLI}<\mathrm{TD}$ \\
\hline & Hutchison et al. [81] & $18(7 ; 9)$ & $24(7 ; 8)$ & $\mathrm{SLI}=\mathrm{TD}$ & $\mathrm{SLI}<\mathrm{TD}$ \\
\hline & Freed et al. [50] & $12(7 ; 9)$ & $23^{\mathrm{a}}(8 ; 3)$ & $\mathrm{SLI}=\mathrm{TD}$ & $\mathrm{SLI}<\mathrm{TD}$ \\
\hline & Alt [72] & $25(7 ; 11)$ & $25(7 ; 10)$ & $\mathrm{SLI}<\mathrm{TD}$ & - \\
\hline & Marini et al. [82] & $32(8 ; 9)$ & $32(8 ; 7)$ & - & $\mathrm{SLI}<\mathrm{TD}$ \\
\hline & Schuchardt et al. [51] & $34^{\mathrm{b}}(9 ; 1)$ & $30(9 ; 1)$ & $\mathrm{SLI}=\mathrm{TD}$ & $\mathrm{SLI}<\mathrm{TD}$ \\
\hline & Lum et al. [53] & $51(9 ; 10)$ & $51(9 ; 10)$ & $\mathrm{SLI}=\mathrm{TD}$ & $\mathrm{SLI}<\mathrm{TD}$ \\
\hline & Henry et al. [58•] & $41(11 ; 6)$ & $88(9 ; 10)$ & $\mathrm{SLI}<\mathrm{TD}$ & $\mathrm{SLI}<\mathrm{TD}$ \\
\hline & Marton et al. [63•] & $22(12 ; 3)$ & $22(12 ; 6)$ & $\mathrm{SLI}<\mathrm{TD}$ & - \\
\hline \multirow[t]{5}{*}{ Inhibition } & Spaulding [55] & $22(4 ; 7)$ & $22(4 ; 8)$ & - & $\mathrm{SLI}<\mathrm{TD}$ \\
\hline & Roello et al. [64•] & $60(5 ; 0)$ & $58(5 ; 0)$ & - & $\mathrm{SLI}<\mathrm{TD}$ \\
\hline & Henry et al. [58•] & $41(11 ; 6)$ & $88(9 ; 10)$ & $\mathrm{SLI}<\mathrm{TD}$ & $\mathrm{SLI}=\mathrm{TD}$ \\
\hline & Epstein et al. [56] & $12(11 ; 5)$ & $22(11 ; 1)$ & $\mathrm{SLI}<\mathrm{TD}$ & - \\
\hline & Marton et al. [63•] & $22(12 ; 3)$ & $22(12 ; 6)$ & $\mathrm{SLI}<\mathrm{TD}$ & - \\
\hline \multirow[t]{3}{*}{ Attentional shifting } & Roello et al. [64•] & $60(5 ; 0)$ & $58(5 ; 0)$ & $\mathrm{SLI}<\mathrm{TD}$ & - \\
\hline & Farrant et al. [57] & $30(5 ; 3)$ & $91(5 ; 1)$ & $\mathrm{SLI}<\mathrm{TD}$ & - \\
\hline & Henry et al. [58•] & $41(11 ; 6)$ & $88(9 ; 10)$ & $\mathrm{SLI}=\mathrm{TD}$ & $\mathrm{SLI}=\mathrm{TD}$ \\
\hline
\end{tabular}

Mean age for each group is reported as years; months

$S L I$ specific language impairment, TD typical language development

${ }^{a}$ Comparison group is children with pragmatic language impairment

${ }^{\mathrm{b}}$ The SLI group includes children with a comorbid dyslexia or learning disability diagnosis

${ }^{\mathrm{c}}$ The range of participant ages is reported because mean age was not reported in the original study using articulatory suppression, which disrupts the ability to use self-directed speech during task completion. Compared to typically developing children, children with SLI performed worse on the executive function measure (the Tower of London task), confirming an executive function deficit for children with SLI. Task performance was worse for both groups of children in the articulatory suppression condition, and the groups' performance was equally affected by articulatory suppression [42]. This pattern of findings suggests that children with SLI use language to mediate executive function performance similarly to children with typical language. In other words, it does not appear to be the case that the poorer executive function performance of children with SLI in this study can be accounted for by their inability to use language to guide response behavior.

Verbal strategy use in SLI was also examined by Eichon et al. [65] by comparing performance on a verbal categorization task in a baseline condition to a condition in which children were instructed to label the category aloud. Children with SLI were less accurate than age-matched typical peers in both the baseline and labeling conditions, indicating a relative deficit in categorization. The labeling condition resulted in no change in accuracy for the typical children, but led to reduced accuracy among children with SLI. Thus, contrary to findings with typically developing children [39, 40], using language as a task strategy may actually be detrimental for children with SLI [65]. 
Many researchers have suggested that documented problems in executive function in SLI may play a causal role in children's language deficits $[66,67]$, but few have directly tested this hypothesis. One interesting line of research in this area has been conducted by Ebert, Kohnert, and colleagues $[68,69]$ using a nonlinguistic cognitive intervention as a possible means of improving language abilities for children with SLI. In two small studies, each with two participants with SLI, these researchers reported that training cognitive skills including auditory memory, processing speed, and sustained attention led to improvements in expressive language abilities [68, 69]. Although these results will need to be replicated with larger samples, improved language skills following treatment targeting executive function provides preliminary support for the potential causal role of executive function in language deficits among children with SLI.

\section{Future Directions}

Despite the increase in attention to the development of executive function in children with SLI, more systematic research is needed to fully understand how executive function and language develop in SLI. First of all, there is a relative dearth of information on how different executive function components may be related to one another within individuals with SLI. We know from work with typically developing adults and children that these components are moderately correlated, but it is unclear whether the same relationship exists for children with SLI. To fill this gap, more research is needed that measures multiple executive function components within the same subjects. Both Henry et al. [58•] and Im-Bolter et al. [62] provide correlations between executive function components and found statistically significant positive relationships between most components. However, these correlations were conducted using the entire sample including typically developing children and children with language impairment combined. Therefore, these results do not address how these components are related in a group of children with SLI alone without the influence of the typically developing children. Future research is needed to examine correlations between executive function components within groups of language impaired children.

Another area that warrants further investigation is the directional relationship between executive function and language abilities in children with SLI. Although some research has addressed this issue, it remains unresolved. Future investigations may use some of the same techniques that have been previously employed to manipulate children's use of language during executive function tasks such as articulatory suppression or instructing children to verbalize; both of which address the role of language in executive function. Likewise, additional research aimed at improving executive function abilities among children with SLI will be helpful in determining the effect of executive function on language abilities. Other methods may also prove useful in addressing this question including longitudinal studies and artificial language learning paradigms. Longitudinal studies that track the growth of language abilities and executive function within language impaired children across time will shed light on how these two skills develop and how the relationship between them may change. Artificial language learning paradigms would offer researchers the ability to test whether children's executive function skills predict their ability to learn language (i.e., grammatical relationships, morphology, vocabulary) by simulating language learning within a controlled laboratory context.

Finally, there are other variables that must be taken into account when measuring executive function that have been largely overlooked in studies of SLI. The first issue is the large variability in executive function abilities associated with age. Because the development of executive function is protracted and continues until early adulthood, it is crucial to consider the effect of age on executive function performance. Comparisons of executive function between children with and without SLI may yield different results depending upon the age of testing. There is often more individual variability in executive function abilities in early childhood during the emergence of these skills, whereas variability may decrease as children age and executive function skills become more stable [70]. Thus, executive function deficits associated with SLI may be more apparent among younger children. Furthermore, due to the effect of age on executive function performance, betweengroup differences may be obscured when studies include a wide range of participant ages because of the resulting within-group variability. Researchers must consider the effect of age on executive function in both the design and interpretation of research comparing children with SLI to typically developing children.

Another variable that has received little attention from researchers examining executive function in SLI is family socioeconomic status (SES), which is positively related to executive function abilities in childhood [71]. Children from families with higher SES, often indexed by parent education, have stronger executive function abilities. Because of the documented relationship between SES and executive function abilities, this variable should be considered when concluding that a group demonstrates an executive function deficit because it may stem from SES discrepancies. Of the studies summarized in Table 1, only four $[48,55,72,73]$ compared or matched SES between SLI and typical language groups. This is especially problematic in the case of SLI because lower parent education is associated with increased likelihood of an SLI diagnosis [74]. Therefore, SES may be a confounding variable in executive function comparisons between children with and without SLI. In future research, researchers should be mindful of this potential confound by matching groups on SES or by 
introducing statistical controls for the influence of SES on executive function abilities in order to establish that executive function deficits are related to SLI and not due to SES discrepancies.

The third variable that is often ignored in comparisons of executive function in children with and without SLI is intelligence. Although some researchers have reported that executive function abilities are independent of general intelligence $[75,76]$, others have found that executive function abilities in childhood are correlated with measures of fluid and crystallized intelligence [34, 77]. Like SES, this presents a potential confound in studies comparing executive function abilities between groups of children with and without SLI. In a recent meta-analysis, Galliant and Spaulding [7] reported that on average children with SLI score 0.69 standard deviations lower than typically developing children on measures of nonverbal intelligence. Thus, it is important for researchers to ensure than any SLI executive function deficits exist separately from disparities in intelligence, which again may be achieved through group matching or through including statistical controls in analyses.

\section{Conclusions}

Based on existing research comparing children with and without SLI on executive function performance, it is apparent that children with SLI demonstrate deficits in at least some executive function components. We argue that it is necessary for future research to move past simply identifying single areas of deficit and to focus instead on testing theories/models of executive function and examining the relationship between executive function deficits and impaired language acquisition. Earlier in this paper, we provided a cautionary note to what we termed Grand Unification Theory approaches to SLI. Having raised this caution, there are advantages of having an overarching theory of executive function to apply to the study of SLI, even if it is unlikely that executive function will prove to be the factor that fully explains the behavioral profile of SLI. The developmental integrative framework model of executive function [38] presented here provides a consideration of executive function that is unified rather than piecemeal. This is likely to promote a more holistic view than the SLI literature to date can provide. This particular framework makes concrete, testable predictions concerning how executive function skills should inter-relate. As such, an integrated view of executive function could help to clarify the nature of these deficits in SLI.

Conversely, the SLI population could provide an interesting and important test case for a developmental integrative framework model of executive function. Data from typically developing children can suggest relationships between skills that may fractionate in the face of a developmental disorder like SLI. Should children with SLI show skill dissociations that their typical peers do not, the proposed dependencies among executive function skills inherent to the model proposed by Garon et al. [38] would be called into question. Whether a developmental integrative framework model can be successfully applied to children with SLI remains to be seen. Moving forward, it is crucial that researchers use models or theories to guide their investigations of executive function in SLI in order to make predictions and interpret results.

Acknowledgments Leah L. Kapa is supported by the National Institute On Deafness And Other Communication Disorders of the National Institutes of Health under Award Number F32DC014188.

\section{Compliance with Ethics Guidelines}

Conflict of Interest Leah L. Kapa and Elena Plante declare that they have no conflict of interest.

Human and Animal Rights and Informed Consent This article does not contain any studies with human or animal subjects performed by any of the authors.

\section{References}

Papers of particular interest, published recently, have been highlighted as:

- Of importance

•. Of major importance

1. Stark RE, Tallal P. Selection of children with specific language deficits. J Hear Disord. 1981;46:114-22.

2. Finlay JCS, McPhillips M. Comorbid motor deficits in a clinical sample of children with specific language impairment. Res Dev Disabil. 2013;34:2533-42.

3. Flapper BCT, Shoemaker MM. Developmental coordination disorder in children with specific language impairment: Co-morbidity and impact on quality of life. Res Dev Disabil. 2013;34:756-63.

4. Miller CA, Kail R, Leonard LB, et al. Speed of processing in children with specific language impairment. J Speech Lang Hear Res. 2001;44:416-33.

5. Botting N, Psarou P, Caplin, et al. Short-term memory skills in children with specific language impairment: the effect of verbal and nonverbal task content. Top Lang Dis. 2013;33:313-27.

6. Lum JAG, Gelgic C, Conti-Ramsden G. Procedural and declarative memory in children with and without specific language impairment. Int J Lang Commun Dis. 2010;45:96-107.

7. Galliant E, Spaulding TJ. With specific language impairment and their typically developing peers on nonverbal cognitive tests: a meta-analysis. J Speech Lang Hear Res. 2014;57:1363-82.

8. Bishop DVM. Ten questions about terminology for children with unexplained language problems. Int J Lang Commun Discord. 2014;49:381-415.

9. Botting N. Non-verbal cognitive development and language impairment. J Child Psychol Psychiatry. 2005;46:317-26.

10. Dethorne LS, Watkins RV. Language abilities and nonverbal IQ in children with language impairment: inconsistency across measures. Clin Linguist Phon. 2006;20:641-58.

11. Plante E. Criteria for SLI: the Stark and Tallal legacy and beyond. J Speech Lang Hear Res. 1998;41:951-7. 
12. Swisher L, Plante E, Lowell S. Nonlinguistics deficits of children with language disorders complicate the interpretation of their nonverbal IQ scores. Lang Speech, Hear ServSch. 1994;25:235-40.

13. Ullman MT, Pierpont EI. Specific language impairment is not specific to language: the procedural deficit hypothesis. Cortex. 2005;41:399-433.

14. Lum JAG, Conti-Ramsden G, Morgan AT, et al. Procedural learning deficits in specific language impairment (SLI): a meta-analysis of serial reaction time task performance. Cortex. 2015;51:1-10.

15. Kail R. A method for studying the generalized slowing hypothesis in children with specific language impairment. J Speech Lang Hear Res. 1994;37:418-21.

16. Miller CA, Leonard LB, Kail RV, et al. Response time in 14-yearolds with language impairment. J Speech Lang Hear Res. 2006;49: 712-28.

17. Morehead DM, Ingram D. The development of base syntax in normal and linguistically deviant children. J Speech Lang Hear Res. $1973 ; 16: 330-52$.

18. Johnston JR, Weismer SE. Mental rotation abilities in languagedisordered children. J Speech Lang and Hear Res. 1983;26:397403.

19. Savich PA. Anticipatory imagery ability in normal and languagedisabled children. J Speech Lang Hear Res. 1984;27:494-501.

20. Kamhi AG, Ward MF, Mills EA. Hierarchical planning abilities in children with specific language impairment. J Speech Lang Hear Res. 1995;38:1108-16.

21. Kamhi AG. Nonlinguistic symbolic and conceptual abilities of language-impaired and normally developing children. J Speech Lang Hear Res. 1981;24:446-53.

22. Hedenius M, Persson J, Tremblay A, et al. Grammar predicts procedural learning and consolidation deficits in children with specific language impairment. Res Dev Disabil. 2011;32:2362-75.

23. Lukács Á, Kemény F. Domain-general sequence learning deficit in specific language impairment. Neuropsychology. 2014;28:472-83.

24. Mayor-Dubois C, Zesiger P, Van der Linden M, et al. Nondeclarative learning in children with specific language impairment: predicting regularities in the visuomotor, phonological, and cognitive domains. Child Neuropsychol. 2014;20:14-22.

25. Lahey M, Edwards J, Munson B. Is processing speed related to severity of language impairment? J Speech Lang Hear Res. 2001;44:1354-61.

26. Plante E, Boliek C, Mahendra N, et al. Right hemisphere contribution to developmental language disorder: neuroanatomical and behavioral evidence. J Commun Disord. 2001;34:415-36.

27. Best JR, Miller PH, Naglieri JA. Relations between executive function and academic achievement from ages 5 to 17 in a large, representative national sample. Learn Individ Differ. 2011;21:327-36.

28. Carlson SM. Developmentally sensitive measures of executive function in preschool children. Dev Neuropsychol. 2005;28:596616.

29. De Luca CR, Wood SJ, Anderson V, et al. Normative data from the Cantab. I: development of executive function over the lifespan. J Clin Exp Neuropsychol. 2003;25:242-54.

30. Brock LL, Rimm-Kaufman SE, Nathanson L, et al. The contributions of 'hot' and 'cool' executive function to children's academic achievement, learning-related behaviors, and engagement in kindergarten. Early Child Res Q. 2009;24:337-49.

31. Lan $\mathrm{X}$, Legare $\mathrm{CH}$, Ponitz $\mathrm{CC}$, et al. Investigating the links between the subcomponents of executive function and academic achievement: a cross-cultural analysis of Chinese and American preschoolers. J Exp Child Psychol. 2011;108:677-92.

32. ST Clair-Thompson HL, Gathercole SE. Executive functions and achievements in school: shifting, updating, inhibition, and working memory. Q J Exp Psychol. 2006;59:745-59.
33. Wiebe SA, Sheffield T, Nelson JM, et al. The structure of executive function in 3-year-olds. J Exp Child Psychol. 2011;108:436-52.

34. Brydges CR, Reid CL, Fox AM, et al. A unitary executive function predicts intelligence in children. Intelligence. 2012;40:458-69.

35. Garavan H, Ross TJ, Murphy K, et al. Dissociable executive function in the dynamic control of behavior: inhibition, error detection, and correction. NeuroImage. 2002;17:1820-9.

36. Fan J, McCandliss BD, Fosella J, et al. The activation of attentional networks. NeuroImage. 2005;26:471-9.

37. Miyake A, Friedman NP, Emerson MJ, et al. The unity and diversity of executive functions and their contributions to complex "frontal lobe" tasks: a latent variable analysis. Cogn Psychol. 2000;41:49 100 .

38. Garon N, Bryson SE, Smith IM. Executive function in preschoolers: a review using and integrative framework. Psychol Bull. 2008;134:31-60.

39. Kirkham NZ, Cruess L, Diamond D. Helping children apply their knowledge to their behavior on a dimension-switching task. Dev Sci. 2003;6:449-76.

40. Marcovitch SE, Miller S. Toddlers benefit from labeling on an executive function search task. J Exp Child Psychol. 2011;108: 580-92.

41. Miyake A, Emerson MJ, Padilla F, et al. Inner speech as a retrieval aid for task goals: the effects of cue type and articulatory suppression in the random task cuing paradigm. Acta Psychol. 2004;115: $123-42$.

42. Lidstone JSM, Meins E, Fernyhough C. Verbal mediation of cognition in children with specific language impairment. Dev Psychopathol. 2012;24:651-60.

43. Gathercole SE, Baddeley AD. Phonological working memory: a critical building block for reading development and vocabulary acquisition? Eur J Psychol Educ. 1993;8:259-72.

44. Kapa LL, Colombo J. Executive function predicts artificial language learning. J Mem Lang. 2014;76:237-52.

45. Novick JM, Hussey E, Teubner-Rhodes S, et al. Clearing the garden-path: improving sentence processing through cognitive control training. Lang Cogn Neurosci. 2013;29(3):186-217.

46. Kemper S, Herman RE, Liu C. Sentence production by young and older adults in controlled contexts. Psychol Sci. 2004;59:220-4.

47. Redmond SM. Conversational profiles of children with ADHD, SLI and typical development. Clin Linguist Phon. 2004;18:107-25.

48. Spaulding TJ, Plante E, Vance R. Sustained selective attention skills of preschool children with specific language impairment: evidence for separate attentional capacities. J Speech Lang Hear Res. 2008;53:16-34

49. Finneran DA, Francis AL, Leonard LB. Sustained attention in children with specific language impairment (SLI). JSpeech Lang Hear Res. 2009;52:915-29.

50. Freed J, Lockton E, Adams C. Short-term and working memory skills in primary school-aged children with specific language impairment and children with pragmatic language impairment: phonological, linguistic, and visuo-spatial aspects. Int J Lang Commun Disord. 2012;47:457-66.

51. Schuchardt K, Bockmann A, Bornemann G, et al. Working memory functioning in children with learning disorders and specific language impairment. Top Lang Disord. 2013;33:298-312.

52. Vugs B, Hendricks M, Cuperus J, et al. Working memory performance and executive function behaviors in young children with SLI. Res Dev Disabil. 2014;35:62-74.

53. Lum JAG, Conti-Ramsden G, Page D, et al. Working, declarative and procedural memory in specific language impairment. Cortex. 2012;48:1138-54.

54. Ziethe A, Eysholdt U, Doellinger M. Sentence repetition and digit span: potential markers of bilingual children with suspected SLI Logoped Phoniatr Vocol. 2013;38:1-10. 
55. Spaulding TJ. Investigating mechanisms of suppression in preschool children with language impairment. J Speech Lang Hear Res. 2010;53:725-38.

56. Epstein B, Shafer VL, Melara RD, et al. Can children with SLI detect cognitive conflict? Behavioral and electrophysiological evidence. J Speech Lang Hear Res. 2014;57:1453-67.

57. Farrant BM, Maybery MT, Fletcher J. Language, cognitive flexibility, and explicit false belief understanding: longitudinal analysis in typical development and specific language impairment. Child Dev. 2012;83:223-35.

58. Henry LA, Messer DJ, Nash G. Executive functioning in children with specific language impairment. J Child Psychol Psychiatry. 2012;53:37-45. This study takes a more integrative approach to the study of executive function in SLI by measuring multiple executive function components. Participants completed verbal and nonverbal measures of working memory, inhibition, and shifting.

59. Ebert KD, Kohnert K. Sustained attention in children with primary language impairment: a meta-analysis. J Speech Lang Hear Res. 2011;54:1372-84.

60.• Vugs B, Cuperus J, Hendricks M, et al. Visuospatial working memory in specific language impairment: a meta-analysis. Res Dev Disabil. 2013;34:2586-97. This meta-analysis addresses the inconsistent results reported across studies of nonverbal working memory in children with SLI. Using effect sizes, the authors conclude that children with SLI have deficits in visuospatial short-term memory and working memory.

61. Duinmeijer I, de Jong J, Scheper A. Narrative abilities, memory and attention in children with a specific language impairment. Int J Lang Commun Dis. 2012;47:542-55. Both attention and working memory were assessed in children with SLI and compared to children with typical language development. Furthermore, children's performance on these executive function measures was correlated with their narrative abilities.

62. Im-Bolter N, Johnson J, Pascual-Leone J. Processing limitations in children with specific language impairment: the role of executive function. Child Dev. 2006;77:1822-41.

63. Marton K, Campanelli L, Scheuer J, et al. Executive function profiles in children with and without specific language impairment. Riv Psicol Appl. 2012;12:57-73. In this study, children with SLI were compared to both age-matched and language-matched children on measures multiple non-verbal executive function components including sustained attention, working memory, and inhibition.

64. Roello M, Ferretti ML, Colonnello V, et al. When words lead to solutions: executive function deficits in preschool children with specific language impairment. Res Dev Disabil. 2015;37:216-22. Preschoolers with SLI were found to perform worse than typically developing age-matched peers on tasks measuring inhibition, shifting, and planning. This study highlights the importance of assessing executive function abilities in early childhood in order to capture group differences in the emergence of these skills.

65. Eichon N, Marton K, Campanelli L, et al. Verbal strategies and nonverbal cues in school-age children with and without specific language impairment (SLI). Int J Lang Commun Dis. 2014;49: 618-30.
66. Leonard LB, Weismer SE, Miller CA, et al. Speed of processing, working memory, and language impairment in children. J Speech Lang Hear Res. 2007;50:408-28.

67. Montgomery JW. Working memory and comprehension in children with specific language impairment: what we know so far. J Commun Disord. 2003;36:221-31.

68. Ebert KD, Kohnert K. Non-linguistic cognitive treatment for primary language impairment. Clin Linguist Phon. 2009;23:647-64.

69. Ebert KD, Rentmeester-Disher K, Kohnert J. Nonlinguistic cognitive treatment for bilingual children with primary language impairment. Clin Linguist Phon. 2012;26:485-501.

70. Huizinga M, Dolan CV, van der Molen MW. Age-related change in executive function: developmental trends and a latent variable analysis. Neuropsychologia. 2006;44:2017-36.

71. Sarsour K, Sheridan M, Jutte D, et al. Family socioeconomic status and child executive functions: the roles of language, home environment, and single parenthood. J Int Neuropsychol Soc. 2011;17: $120-32$.

72. Alt M. Visual fast mapping in school-aged children with specific language impairment. Top Lang Dis. 2013;33:328-46.

73. Petruccelli N, Bavin EL, Bretherton L. Children with specific language impairment and resolved late talkers: working memory profiles at 5 years. J Speech Lang Hear Res. 2012;55:1690-703.

74. Tomblin JB, Smith E, Zhang X. Epidemiology of specific language impairment: prenatal and perinatal risk factors. J Commun Disord. 1997;30:325-44.

75. Ardila A, Pineda D, Rosselli M. Correlation between intelligence test scores and executive function measures. Arch Clin Neuropsychol. 2000;15:31-6.

76. Lehto JE, Juujärvi P, Kooistra L, et al. Dimension of executive functioning: evidence from children. Br J Dev Psychol. 2003;21: 59-80.

77. Arffa S. The relationship of intelligence to executive function and non-executive function measures in a sample of average, above average, and gifted youth. Arch Clin Neuropsychol. 2007;22: 969-78.

78. Dispaldro M, Leonard LB. Corradi, et al. Visual attentional engagement deficits in children with specific language impairment and their role in real-time language processing. Cortex. 2013;49: 2126-39.

79. Montgomery JW, Evans JL, Gillam RB. Relation of auditory attention and complex sentence comprehension in children with specific language impairment: a preliminary study. Appl Psycholinguist. 2009;30:123-51.

80. Montgomery JW. Role of auditory attention in the real-time processing of simple grammar by children with specific language impairment: a preliminary investigation. Int $\mathrm{J}$ Lang Commun Dis. 2008:43:499-527.

81. Hutchison E, Bavin E, Efron D, Sciberras E. A comparison of working memory profiles in school-aged children with specific language impairment, attention deficit/hyperactivity disorder, comorbid SLI and ADHD and their typically developing peers. Child Neuropsychol. 2012;18:190-207.

82. Marini A, Gentili C, Molteni M, et al. Differential verbal working memory effects on linguistic production in children with specific language impairment. Res Dev Disabil. 2014;35:3534-42. 